\title{
Twice Neglected? Neglected Diseases in Neglected Populations
}

\author{
Ethel Weld ${ }^{1}$, Catriona Waitt ${ }^{2}$, Karen Barnes ${ }^{3}$, and FACUNDO GARCIA-BOURNISSEN ${ }^{4}$ \\ ${ }^{1}$ Johns Hopkins University School of Medicine \\ ${ }^{2}$ University of Liverpool Institute of Translational Medicine \\ ${ }^{3}$ Division of Clinical Pharmacology, Department of Medicine, University of Cape Town, \\ Cape Town, South Africa. \\ ${ }^{4}$ National Research Council (CONICET)
}

September 25, 2021

\begin{abstract}
It is unfortunately true that clinicians lack the necessary evidence to know how to use medications properly in large sections of the population, and we do not have optimal drugs to use in many Neglected Tropical Diseases (NTDs). NTD's often disproportionately affect neglected populations such as children and pregnant women. As reliable access to safe, effective preventives and treatments can break the cycle of poverty, illness, and ensuing debility that further perpetuates poverty, it is of paramount importance to investigate and develop new medicines for neglected populations suffering from NTDs. Furthermore, there is not only a need to develop and evaluate novel therapies, but also to ensure that these are affordable, available, and adapted to the communities who need them. With this editorial, the British Pharmacological Society hereby launches a call for high-quality articles focusing on NTDs in special populations, to facilitate the reversal of this dual neglect.
\end{abstract}

\section{Editorial}

(To accompany the call for papers for the Twice Neglected series)

BJCP

\section{Twice Neglected? Neglected Diseases in Neglected Populations}

\section{The Problem: Neglected diseases and why they matter}

Over a billion people worldwide experience morbidity and mortality from conditions for which there are few evidence-based treatments. Neglected tropical diseases (NTDs) impose a devastating human, social and economic burden on predominantly impoverished populations, mainly in tropical and subtropical areas where the most vulnerable, marginalized populations of the world live. ${ }^{1,2}$ The World Health Organization (WHO) definition of NTDs includes the phrase "ancient diseases of poverty", which though poetic leaves out emerging infectious threats and diseases such as malnutrition, intoxications due to poorly regulated industrial activity (e.g. lead, mercury, pesticides exposures) and many other conditions (See Table 1) which disproportionately affect the poor and disenfranchised, and about which too little is known, or done.

Over recent decades, evidence-based medicine has been accepted as the standard upon which to base high quality treatment guidelines. Contrasted with this is the problematic reality: we lack the necessary evidence to know how to use medications properly in large sections of the population, and we do not have optimal drugs to use in many NTDs. Moreover, these conditions often disproportionately affect neglected populations such as children and pregnant women. As reliable access to safe, effective preventives and treatments can break the cycle of poverty, illness, and ensuing debility that further perpetuates poverty, it is of paramount importance to investigate and develop new medicines for neglected populations suffering from NTDs. Furthermore, 
there is not only a need to develop and evaluate novel therapies, but also to ensure that these are affordable, available, and adapted to the communities who need them.

\section{What harm is caused by this neglect?}

The examples of harm from this double neglect are many. Possibly due to their chronic nature (e.g. many NTDs do not lead to high acute mortality, but to chronic, debilitating disease), and geographical distance from high-income countries to populations with NTDs, NTDs are perceived as infrequent and their complications and long-term sequelae of little consequence. However, even if many NTDs are not acutely deadly, (e.g. Chagas disease, cutaneous leishmaniasis, soil-transmitted nematodes) they have large impacts on individual well-being and capabilities, local economies and health systems, and have direct bearing on human flourishing (e.g. as measured by the key metric the United Nations Human Development Index)). ${ }^{3,4}$ On the other hand, some NTDs also have high mortality, particularly concentrated in neglected populations; for example, the mortality and morbidity burdens for malaria, TB meningitis and disseminated TB are centered in children, and Ebola is almost uniformly fatal both to pregnant women and to their neonates, if left untreated. ${ }^{5} \mathrm{~A}$ large portion of individuals worldwide who die each year from diarrhoeal disease are children, particularly with underlying malnutrition. ${ }^{6,7}$ It is well documented that there are often altered physiological factors that can affect drug pharmacokinetics, leading in many cases to related safety and efficacy concerns in vulnerable populations, such as those with prevalent co-morbidities-obese adults, pregnant women and young children, particularly those with malnutrition. ${ }^{8-10}$

\section{What is a Neglected Tropical Disease?}

The WHO lists a diverse set of twenty diseases or disease groups as priority 'neglected tropical diseases' See Table $1 .{ }^{11}$ Others have expanded the list to include 40 conditions unified in their ability to perpetuate poverty and cause chronic debility. ${ }^{12}$ Control of these neglected diseases is variable - they range from almost eliminated (e.g. dracunculiasis) to precipitously expanding in both prevalence and range (e.g. dengue). ${ }^{13}$ They have a singular commonality: their devastating impact on impoverished communities. However, the list is not exhaustive! Other situations exist where a dearth of evidence results in uncertainty about clinical management, including drug-resistant pathogens such as tuberculosis and malaria, and emerging infectious diseases such as melioidosis, hantavirus, filoviruses, and Zika virus.

\section{What is a Neglected Population?}

For our purposes, a 'neglected population' is 'a population in whom there is insufficient evidence to inform the safe and effective use of medication'. Clinical trials often stipulate stringent eligibility criteria which are not reflective of the real-life clinical population who require treatment. Many clinical and demographic factors impact on disease burden and drug disposition, and whilst it is increasingly recognized that it is ethically imperative to study drugs in the populations in whom they are to be used, this is rarely the case in practice. ${ }^{14}$ An increasing body of work argues for and exemplifies the study of drugs in pregnant and lactating women and their infants, children and adolescents, ${ }^{15,16}$ but even in these there remains an unacceptable delay between the time of drug licensing and the availability of data to inform safe and effective use in such populations. ${ }^{17}$ Where intersecting characteristics exist that render an individual 'complex' - such as young, malnourished children with malaria or elderly individuals with prevalent co-morbid conditions - clinicians are forced to make a 'best guess' as to the appropriate drug treatment for that individual, perhaps leading to segments of the population that are thrice neglected (the next vanguard, once the twice neglected receive their fair due in research).

\section{Why are these diseases and populations neglected?}

Who is doing the neglecting? The neglect in this case extends across multiple domains, from the research enterprise (pharmaceutical manufacturers, funding bodies, academic researchers, regulatory bodies, scientific literature publishing enterprise) to governments, and across the public and private sectors. There are four agents of neglect that we shall consider here: financial, geospatial, protectionist, and regulatory. These synergize with each other and create overlapping conditions that foster more neglect. Many neglected diseases 
and conditions occur in under-resourced, underserved, settings with inadequate access to medical research and care, perpetuating their neglect. The financing of research on these topics has often been relegated to non-governmental, non-corporate entities and public-private partnerships that are often external to the affected area, with a focus on a single disease; this approach is limited as it does not contend with the reality that there are often multiple overlapping medical and socioeconomic conditions at play in a given region that intersect differently in different populations. ${ }^{13}$ In effectively leveraging the Global Fund for AIDS, tuberculosis and malaria, there exists a tension between the benefits of focusing on specific diseases and the reality that special populations affected by these diseases are also affected by other overlapping povertyrelated diseases. Drug donations from pharmaceutical manufacturers have been a key element in Mass Drug Administration campaigns to address filariasis, helminthic infection, and other parasitic diseases, ${ }^{18}$ but donations from Industry of other kinds (e.g., research support and infrastructure) ${ }^{19}$ could amplify this positive impact from the private sector so that NTDs can be studied in special populations, including incorporation of pharmacovigilance systems and assessment of overall societal impacts.

Geospatial inequity foments research neglect too; nations in the global South are often left out of initiatives to improve health that disproportionately serve the global North. The current COVID-19 pandemic provides a searing example of why the approach to infectious illness must be global and equitable. Out of the two billion doses of SARS-CoV2 vaccine that had been administered worldwide by August of 2021, only 1.4\% of them were delivered in low-income countries. ${ }^{20}$ Uneven and inadequate vaccine distribution to regions where COVID-19 cases are surging offers an excellent opportunity for the virus to multiply and mutate within innumerable human hosts, with obvious downstream effects for all countries irrespective of their level of resources. Furthermore, tropical diseases are anticipated to become global problems, with climate change. ${ }^{21}$ With a warming planet, dengue has become widespread, leishmaniasis is now endemic in Italy, Chagas in the Southern U.S.A. ${ }^{22}$ An inversion or epidemiological shift is occurring: as diseases of affluence come to the cities of the global South, neglected diseases of poverty may come to the global North. Clearly, decolonizing globally-minded research attitudes is needed in the face of these new waves.

Even once funding hurdles are cleared, investigators with the best intentions to do rigorous research on neglected conditions in neglected populations are often thwarted by regulatory barriers. Both global and country-specific research ethics committees and regulatory bodies can be hesitant about performing research on populations who are perceived to be vulnerable (and thus, in turn, rendered even more vulnerable by being left out of research by these roadblocks). Furthermore, regulatory barriers have frequently been construed, and perhaps misconstrued, as non-negotiable and static absolutes rather than as principles that dynamically parallel evolving ethical stances.

A lack of financial investment in research on the safety and efficacy of drugs in special populations has at times been nominally justified by a desire to protect the "vulnerable" from harm. This has only compounded the research neglect of key populations suffering undue burdens of NTDs. Too long has a misplaced belief in protecting so-called vulnerable populations "protected them to death" by creating a knowledge void around their treatment and care. ${ }^{23}$ In essence, imagining these populations to be special and in need of protection has engendered their neglect in research. Lastly, the choicelessness of individuals affected by NTDs in underresourced areas serves as perhaps the starkest counterpoint and rebuttal to the animus-fueled assertion that these conditions are inevitable, acceptable, and unworthy of study because they arise from personal or behavioral failings.

\section{Why do neglected populations present a particular challenge?}

With non-neglected conditions, sample size and inclusion criteria usually mean that data on highly refined subgroups of patients emerges from the research (i.e., the different considerations for initiating a calcium channel blocker as opposed to an ACE inhibitor for hypertension). However, for neglected conditions, a further neglect emerges - the neglect of patient complexity. The study of problems that disproportionately affect the poor can be needlessly homogenizing - ignoring individual patient characteristics which better funded conditions incorporate in their evidence-based solutions. 
Another aspect of neglect hinges on the assumption of commensurability of one population for another (i.e., the idea that it is possible to extrapolate efficacy, safety and pharmacokinetic data from one population, e.g. healthy European males in their fifties to another, e.g. pregnant African women in their twenties). This assumption does not hold true in many situations, particularly where there are differences in physiology, pharmacogenetics or intercurrent disease states (e.g., renal clearance differences in pregnancy or early childhood). In cases where the research neglect relates to geopolitical or socioeconomic factors (refugees, prisoners, nomadic groups, etc.), there may be an indirect impact on pharmacokinetic parameters through intermediaries such as adherence and instability of access to food or care, but overall, it may be reasonable to extrapolate pharmacokinetic parameters from one population to another. Dietary factors may differ substantially across populations, so medicines tested in North America or Europe and then brought to Africa or Asia can encounter different dietary patterns which may affect absorption and permeability of the medicine, depending on the compound. Similarly, pharmacogenetic differences may result in clinically significant differences in drug exposure. ${ }^{24}$

Another consideration surrounds the question of when can we extrapolate safety and efficacy of repurposed drugs from one condition to another poorly understood or neglected condition, and when can we not? This can be done well in some conditions, as in the repurposing of rifamycins for leprosy prevention, and less well, as in the effort to take a "kitchen sink" approach to COVID-19 treatment based only on limited preclinical data but no rational clinical pharmacologic basis. An example of this is seen with highly protein-bound drugs' $\mathrm{EC}_{50}$ against SARS-COV2 that cannot be clinically achieved in humans using safe doses. ${ }^{25}$ A more rational stepwise model-informed drug repurposing approach has been proposed, integrating preparatory, reactive, and retrospective action in response to new pathogens. ${ }^{26,27}$ In essence, situations of desperation call for more high-quality rational science, and not less. There is strong potential for meta-analyses of pooled individual patient data (IPD) to speed up the answering of key questions, provided they adjust for relevant heterogeneity (e.g. disease severity, dose and other drivers of drug exposure). Overall, there is an ethical imperative not to shift risk from well-controlled, carefully monitored small studies to widespread uninformed use in the general population which may be considered to amount to (often poorly controlled) experimentation. However, the latter describes the reality of how many drugs for neglected tropical diseases have been developed for special populations.

The regulatory aspects of study of drugs in special populations are complex. In some settings, strong regulatory advice that drug manufacturers study drugs in special populations such as children or orphan conditions such as XDR-TB has been incentivised with certain benefits, such as vouchers and patent extensions. There has been some criticism of this, particularly as it has not led to improvement in price or availability for many drugs that were given such incentives (e.g. miltefosine, benznidazole and several other drugs for NTDs remain difficult to obtain, prohibitively expensive or both in North America, after the pharmaceutical companies that registered them obtained hundreds of millions of dollars in benefits). ${ }^{28}$ Some have argued that the situation of inequitable study of special populations calls for a "carrot and stick approach", where not just incentives, but strict enforcement mechanisms with punitive measures attached are needed. The passage of the Pediatric Research Equity Act (PREA) and the Best Pharmaceuticals for Children Act (BPCA), introduced as part of the FDA Safety and Innovation Act (FDASIA), took this approach. ${ }^{29,30}$ It was accompanied by guidance on how to comply with the acts, which delineated the need for study in four main age groups of children: neonates, infants, children, and adolescents. The PREA (viewed as the "stick") requires that pharmaceutical companies submit a plan for a pediatric study with each new drug application. The drug can be launched in adults even if not yet tested in children, but the sponsor must submit its assessments in children by a certain final due date. BPCA (viewed as the "carrot") grants sponsors who conduct pediatric studies of their products six months of market exclusivity to the company for that pediatric product. Over 85 exclusivity determinations have been granted by the FDA since September of 2007. Similar "carrot and stick" initiatives do not yet exist for pregnant, obese, or elderly individuals even though they carry disproportionately high burdens of some diseases.

\section{How can novel treatments be safely and ethically investigated?}


The clinical evaluation of a potential treatment in these 'complex' or 'neglected groups' requires first a balancing of risks and benefits, including whether or not there is better data for alternative treatments in the vulnerable sub-population. (An example of harm caused by a skewed balancing of these risks was treating malaria in pregnant women and young children with chloroquine long past the point of widespread chloroquine resistance, because it was perceived as safe for the vulnerable, even though these groups were also at the highest risk of the potentially severe consequences of failed malaria treatment.) It also requires innovative approaches, including partnership between clinical pharmacologists and pharmacometricians. Physiologicallybased pharmacokinetic modelling uses a 'bottom-up' approach based on knowledge of cellular processes to predict drug exposure within a specific population. Models can be adapted to encompass characteristics such as nutritional status, hepatic or renal dysfunction, pregnancy, concurrent administration of interacting medications and other factors which may render a population 'neglected' ${ }^{31-33}$ This can provide a starting point for clinical trials to confirm the predicted dosing strategies in vulnerable and neglected patient groups. Optimal design theory uses mathematical approaches to predict the ideal sampling schedule for the most efficient study design. This enables the minimum number of participants to be included with the least invasive sampling schedule in order to yield the highest quality information. ${ }^{34,35}$ Population pharmacokinetic modelling explores sources of variability between individuals, and adds to the understanding of how different 'neglected' characteristics may impact on drug exposure and thereby on the appropriate dosing schedules to ensure safe, effective treatment. ${ }^{36}$ Combinations of these techniques have the potential to yield high quality, efficient clinical studies and advance prompt understanding of sources of variability in drug exposure and thus potentially efficacy and safety, particularly in these difficult-to-study groups. ${ }^{37}$

\section{How can new drugs for NTDs be developed?}

The Drugs for Neglected Diseases Initiative (DNDi) was created in response to the frustration of clinicians and the desperation of patients faced with medicines that were ineffective, unsafe, unavailable, unaffordable, or that had never been developed at all. DNDi embraces the power of innovation, open science, partnerships, and advocacy to find solutions to a great injustice: the lack of medicines for life-threatening diseases that disproportionately impact poor and marginalized people. ${ }^{38}$ Through harnessing scientific advances in drug discovery, technologies to improve the efficiency and accelerate the pace of the R\&D process, including artificial intelligence (AI)-driven drug discovery tools, novel imaging, diagnostic, and clinical trial design and operations technologies, and AI-driven data analysis are being employed. Evaluation of a range of strategies and compounds, including fast-evolving techniques such as the use of host-targeted therapies or development of specific monoclonal antibodies alongside repurposing of older agents aims to increase the therapeutic repertoire available for evaluation in clinical trials. Furthermore, efforts to develop drugs for NTDs must involve engaging members of the affected communities, and the control arms of randomized controlled trials of new drugs must at least include the existing national standard of care. Similar models have been used in partnerships such as the Medicines for Malaria Venture, Gavi (the vaccine alliance) and the COVID-19 Clinical Research Coalition.

\section{Examples of best practice in the study of Special Populations}

The British Pharmacological Society has been at the forefront of driving a call for sex to be considered an experimental variable in research $;^{39}$ now we move one step further and call for the generation of relevant evidence for the management of the most neglected diseases, occurring in the most neglected populations (i.e. "Twice-neglected" populations).

The NIH Policy on sex as an experimental variable includes an easy-to-remember four Cs framework that is as relevant for neglected diseases and populations: Consider : when designing studies, either take the neglected population and/or disease into account or explain why you haven't; Collect : tabulate data from neglected populations that take into account differences from the general population; Characterize : analyze data in such a way that differences by population can be detected; Communicate : report and publish data about neglected diseases and neglected populations. ${ }^{40}$

Through the "Twice Neglected" series, the British Pharmacological Society will encourage the conduct and 
facilitate the dissemination of high-quality articles that will reduce the neglect of diseases of poverty in complex populations. Progress requires multi-disciplinary collaboration, knowledge-transfer and a visionary, ethics-rooted approach

In times past, some major drug discoveries occurred almost fortuitously (e.g., Alexander Fleming's discovery of penicillin) but solutions to NTDs, especially when impacting neglected vulnerable populations, will not be discovered without the deliberate, targeted focus of the research community, involving strong partnerships between community, industry, academia and clinicians, with multi-disciplinary collaboration between cuttingedge technologies and a vision which prioritises alleviation of human suffering. Clinical pharmacology is in its essence cross-disciplinary, and brings together many of the key skills and actors required to transform such a vision into a reality with disruptive, silo-colliding thinking and efforts. Neglect results from persistent inattention, and as a community of pharmacologists we hereby launch this call for papers to draw awareness to these conditions and special populations throughout the processes of drug discovery and evaluation, to facilitate knowledge transfer, to stimulate dialogue and interaction, and ultimately to be part of the solution to this global problem. May it be received as a clarion call to arms.

Table 1. Selected Neglected Tropical Diseases (NTDs)

WHO-Defined NTDs: (REF) Buruli ulcer (Mycobacterium ulcerans) Chagas disease Dengue and Chikungunya Dracunculias

\section{References}

1. Mitra AK, Mawson AR. Neglected Tropical Diseases: Epidemiology and Global Burden. Trop Med Infect Dis. 2017;2(3).

2. Casulli A. New global targets for NTDs in the WHO roadmap 2021-2030. PLoS Negl Trop Dis. 2021;15(5):e0009373.

3. Wilsher E, Jane. The impact of Neglected Tropical Diseases, and their associated stigma, on people's basic capabilities. Durham, UK2011.

4. Nations U. UN Human Development Report. The Next Frontier: Human Development and the Anthropocene 2020 .

5. Sayres L, Hughes BL. Contemporary Understanding of Ebola and Zika Virus in Pregnancy. Clin Perinatol. 2020;47(4):835-846.

6. Collaborators GDD. Estimates of the global, regional, and national morbidity, mortality, and aetiologies of diarrhoea in 195 countries: a systematic analysis for the Global Burden of Disease Study 2016. Lancet Infect Dis. 2018;18(11):1211-1228.

7. Tickell KD, Sharmin R, Deichsel EL, et al. The effect of acute malnutrition on enteric pathogens, moderate-to-severe diarrhoea, and associated mortality in the Global Enteric Multicenter Study cohort: a post-hoc analysis. Lancet Glob Health. 2020;8(2):e215-e224.

8. Hazenberg P, Navaratnam K, Busuulwa P, Waitt C. Anti-Infective Dosing in Special Populations: Pregnancy. Clin Pharmacol Ther. 2021;109(4):977-986.

9. Maharaj AR, Wu H, Zimmerman KO, et al. Pharmacokinetics of Ceftazidime in Children and Adolescents with Obesity. Paediatr Drugs. 2021.

10. Ferro A. Paediatric prescribing: why children are not small adults. Br J Clin Pharmacol. 2015;79(3):351353.

11. WHO. Ending the neglect to attain the Sustainable Development Goals: A road map for neglected tropical diseases 2021-20302020. 
12. Hotez PJ, Aksoy S, Brindley PJ, Kamhawi S. What constitutes a neglected tropical disease? PLoS Negl Trop Dis. 2020;14(1):e0008001.

13. Reed SL, McKerrow JH. Why Funding for Neglected Tropical Diseases Should Be a Global Priority. Clin Infect Dis. 2018;67(3):323-326.

14. Weld ED, Bailey TC, Waitt C. Ethical issues in therapeutic use and research in pregnant and breastfeeding women. Br J Clin Pharmacol. 2021.

15. Gupta A, Hughes MD, Garcia-Prats AJ, McIntire K, Hesseling AC. Inclusion of key populations in clinical trials of new antituberculosis treatments: Current barriers and recommendations for pregnant and lactating women, children, and HIV-infected persons. PLoS Med. 2019;16(8):e1002882.

16. Eke AC, Olagunju A, Momper J, et al. Optimizing Pharmacology Studies in Pregnant and Lactating Women Using Lessons From HIV: A Consensus Statement. Clin Pharmacol Ther. 2021;110(1):36-48.

17. Colbers A, Mirochnick M, Schalkwijk S, Penazzato M, Townsend C, Burger D. Importance of Prospective Studies in Pregnant and Breastfeeding Women Living With Human Immunodeficiency Virus. Clin Infect Dis. 2019;69(7):1254-1258.

18. Bradley M, Taylor R, Jacobson J, et al. Medicine donation programmes supporting the global drive to end the burden of neglected tropical diseases. Trans R Soc Trop Med Hyg. 2021;115(2):136-144.

19. Gutierrez MM, Pillai G, Felix S, et al. Building Capability for Clinical Pharmacology Research in Sub-Saharan Africa. Clin Pharmacol Ther. 2017;102(5):786-795.

20. Global COVID Vaccination Rates, Our World in Data2021.

21. Booth M. Climate Change and the Neglected Tropical Diseases. Adv Parasitol. 2018;100:39-126.

22. Hotez PJ. The rise of neglected tropical diseases in the "new Texas". PLoS Negl Trop Dis. 2018;12(1):e0005581.

23. Gomes MF, de la Fuente-Núñez V, Saxena A, Kuesel AC. Protected to death: systematic exclusion of pregnant women from Ebola virus disease trials. Reprod Health. 2017;14(Suppl 3):172.

24. Mthiyane T, Millard J, Adamson J, et al. -Acetyltransferase 2 Genotypes among Zulu-Speaking South Africans and Isoniazid and. Antimicrob Agents Chemother. 2020;64(4).

25. Schmith VD, Zhou JJ, Lohmer LRL. The Approved Dose of Ivermectin Alone is not the Ideal Dose for the Treatment of COVID-19. Clin Pharmacol Ther. 2020;108(4):762-765.

26. Siccardi M, Schapiro J, Di Perri G, Back DJ. The challenging pathway towards the identification of SARS-CoV-2/COVID-19 therapeutics. J Antimicrob Chemother. 2020;75(9):2381-2383.

27. Dodds M, Xiong Y, Mouksassi S, et al. Model-informed drug repurposing: A pharmacometric approach to novel pathogen preparedness, response and retrospection. Br J Clin Pharmacol. 2021.

28. Shahriar AA, Alpern JD. Antiparasitic Drugs in the United States-Two Roads to High Prices. Front Sociol. 2020;5:540478.

29. US Food and Drug Administration. Pediatric Research Equity Act of 2003.

30. Qualifying for Pediatric Exclusivity Under Section 505A of the Federal Food, Drug, and Cosmetic Act.

31. Johnson TN, Abduljalil K, Nicolas JM, et al. Use of a physiologically based pharmacokineticpharmacodynamic model for initial dose prediction and escalation during a paediatric clinical trial. Br $\mathrm{J}$ Clin Pharmacol. 2021;87(3):1378-1389.

32. Stader F, Courlet P, Kinvig H, et al. Effect of ageing on antiretroviral drug pharmacokinetics using clinical data combined with modelling and simulation. Br J Clin Pharmacol. 2021;87(2):458-470. 
33. Xu R, Tang H, Chen L, Ge W, Yang J. Developing a physiologically based pharmacokinetic model of apixaban to predict scenarios of drug-drug interactions, renal impairment and paediatric populations. Br $\mathrm{J}$ Clin Pharmacol. 2021;87(8):3244-3254.

34. Simpson JA, Jamsen KM, Price RN, et al. Towards optimal design of anti-malarial pharmacokinetic studies. Malar J. 2009;8:189.

35. Standing JF. Understanding and applying pharmacometric modelling and simulation in clinical practice and research. Br J Clin Pharmacol. 2017;83(2):247-254.

36. Holford N. Clinical pharmacology $=$ disease progression + drug action. Br J Clin Pharmacol. 2015;79(1):18-27.

37. Tsamandouras N, Rostami-Hodjegan A, Aarons L. Combining the 'bottom up' and 'top down' approaches in pharmacokinetic modelling: fitting PBPK models to observed clinical data. Br J Clin Pharmacol. 2015;79(1):48-55.

38. Drugs for Neglected Tropical Diseases initiatives Strategic Plan 2021-2028. https://dndi.org/wpcontent/uploads/2021/03/DNDi-StrategicPlan-2021-2028.pdf.

39. Gogos A, Langmead C, Sullivan JC, Lawrence AJ. The importance of sex differences in pharmacology research. Br J Pharmacol. 2019;176(21):4087-4089.

40. NIH. NIH Policy on Sex as a Biological Variable. https://orwh.od.nih.gov/sex-gender/nih-policy-sexbiological-variable. 